\title{
Performance evaluation of precision nanopositioning devices caused by uncertainties due to tolerances using function approximation moment method
}

\author{
J. S. Huh, ${ }^{\text {a) }}$ K. H. Kim, D. W. Kang, D. G. Gweon, and B. M. Kwak \\ Engineering system and design Laboratory, Department of Mechanical Engineering, Korea Advanced Institute \\ of Science \& Technology, 373-1 Guseong-Dong, Yuseong-Gu, ME5004, Daejeon 305-701, Korea
}

(Received 31 October 2005; accepted 5 December 2005; published online 19 January 2006)

Precision nanopositioning is an important technology in industry and requires tight design specifications. Tolerances, although very small, are allocated in all dimensions of structures at devices and are understood as sources of performance variations. In this research, we aim to study detail influence of tolerances on various system response functions of a precision stage, especially parasitic motion and resonant frequencies. A function approximation moment method (FAMM) is developed and applied to study it. The variations are mathematically expressed as their statistical moments and the probabilities of satisfaction are obtained as a result of the FAMM. A finite element model of the target stage, which is nonmonolithic, is generated and verified with basic measurements. The possible initial deformation after assembly is found by the formulation of minimizing strain energy. With this model, the FAMM is used to estimate the statistical moments and probability density functions of the performance functions. The calculated results facilitate understanding of the characteristics of the stage in terms of probability. The inevitable mismatch, even under a small tolerance, is found to cause a large parasitic motion and should be considered in the design and manufacturing process. () 2006 American Institute of Physics.

[DOI: $10.1063 / 1.2162750]$

\section{INTRODUCTION}

Nanopositioning technology is getting more important in recent engineering fields such as scanning probe microscopy, lithography, precision measurement, and micromachining. They require high displacement resolution, wide motion range, and high speed response without parasitic motion. Most of the stages developed are composed of guiding and magnifying mechanisms such as flexure hinge and leaf spring, and actuators such as a piezoelectric transducer actuator, voice-coil motors, and linear motors. Since tolerances are inevitable in all dimensions of the flexure mechanisms like other engineering structures, the performances may not be up to the requirements, as in an ideal situation. Smith $e t$ $a l .{ }^{1}$ demonstrated that a drive system could cause significant parasitic motion due to manufacturing tolerances even in very stiff systems. Ryu and Gweon ${ }^{2}$ have studied their influences on the motion of a monolithic flexure hinge mechanism. They have used a parametric study and a worst case study where the maximum motion error among the full factorial, $2^{N}$ computed errors, is found. However, these types of methods could not present mathematically strict effects of system uncertainties when compared to probabilistic approaches. Various probabilistic methodologies ${ }^{3-5}$ have been developed and applied to a diversity of engineering fields. Those methods have advantages and disadvantages in terms of application scope, accuracy, and computational cost.

\footnotetext{
${ }^{a)}$ Electronic mail: jshuh@khp.kaist.ac.kr
}

The goal is to analyze the variations of performance functions of the $X Y$ stage developed by Kang et al., ${ }^{6}$ especially parasitic motion and resonant frequencies related to response speed. The stage is composed of a flexure (leafspring) guide of a double compound linear spring ${ }^{2}$ and voicecoil motor (VCM) actuator. An assembly structure is adopted in this system instead of a monolithic stage because of difficulties of making a thin monolithic leaf spring. Therefore, tolerances of the parts may accumulate and degrade the performance of the $X Y$ stage. To analyze the initial deformation of the assembled stage, a finite element (FE) model validated with measurements is generated. With this model, a function approximation moment method (FAMM) proposed in this paper is utilized to calculate the statistical moments of the system response functions and to estimate their probability density functions. Given design requirements, probabilities of satisfaction are easily calculated. The information allows designers to understand quantitatively all the characteristics of the $X Y$ stage.

\section{METHODOLOGY}

\section{A. Moment method}

The variations of a performance function, $g(\mathbf{x})$, may mathematically be expressed by the statistical moments of $g(\mathbf{x})$ and the $k$ th moment is defined as follows: 


$$
\begin{aligned}
E\left\{g^{k}\right\}= & \int_{-\infty}^{\infty} \cdots \int_{-\infty}^{\infty}\left[g\left(x_{1}, x_{2}, \ldots, x_{N}\right)\right. \\
& \left.-\mu_{g}\right]^{k} \prod_{i=1}^{N} \phi_{i}\left(x_{i}\right) d x_{1} d x_{2} \cdots d x_{N},
\end{aligned}
$$

where $\mu_{g}$ is the mean of $g(\mathbf{x})$, and $\phi_{i}$ is the probability density function of random variable $x_{i}$ for $i=1$ to $N$, which is assumed uncorrelated. Tolerances of design variables are a typical class of independent random variables. Once information of several moments from low orders is given, a probability density function can be empirically obtained. Various moment methods ${ }^{4}$ have been developed and may be categorized depending on the order of moments included. The moment method adopted here is the fourth-moment method. It calculates the first four moments of $g(\mathbf{x})$, that is, mean, standard deviation (S.D.), skewness, and kurtosis, and utilizes the Pearson system ${ }^{7}$ to estimate the probability density function. In moment methods, the biggest issue is how to efficiently and accurately calculate the first four moments.

\section{B. Function approximation moment method (FAMM)}

Generally, a system response function, $g(\mathbf{x})$, is not given as an explicit function such as polynomial expression and it is almost impossible to integrate the $k$ th order moment analytically. Therefore, FAMM is to estimate the first four moments of $g(\mathbf{x})$ using a suitable quadratic interpolating polynomial, $\bar{g}(\mathbf{x})$, which approximates $g(\mathbf{x})$ over a canonical experimental region and then to identify the probability density function of $g(\mathbf{x})$. The overall procedure of FAMM is summarized as follows:

Step 1. Considering the one-dimensional problem about each random variable $x_{i}$, for $i=1$ to $N$, the positions of three distinct points to determine the coefficients of a quadratic polynomial are optimized by three error-minimizing conditions, as suggested below.

Step 2. An $N$-orthotope is defined by these points as a canonical experimental region for defining a suitable polynomial, $\bar{g}(\mathbf{x})$.

Step 3. On this constructed region, a design of experiments with $(N+1)(N+2) / 2$ function evaluations is performed, and the coefficients of $\bar{g}(\mathbf{x})$ are uniquely determined.

Step 4. The first four moments of $\bar{g}(\mathbf{x})$ are easily obtained as the estimates for $g(\mathbf{x})$.

Step 5. Identify the proper type of the Pearson system and obtain the probability of failure or satisfaction.

\section{Three error-minimizing conditions and the canonical experimental region}

In order to construct the canonical experimental region for function approximation, one must consider a onedimensional integration problem. Positions of three distinct points $(l, m, n)$ determine the coefficients of an interpolating polynomial of order $2, \bar{g}(x)$, as an approximate function. It is necessary to optimize them for better accuracy. It might well be reasonable that the cubic polynomial is expected to approximate more closely a system response function than the quadratic polynomial. In this paper, three error-minimizing conditions are proposed, as explained below, and require that the function $\bar{g}(x)$ best approximates an arbitrary polynomial function of order 3 from the viewpoint of the moments. Therefore, the response function $g(x)$, assumed as a cubic polynomial, as

$$
g(x)=a x^{3}+b x^{2}+c x+d
$$

and the interpolating function, $\bar{g}(x)$, is quadratic as

$$
\bar{g}(x)=\bar{a} x^{2}+\bar{b} x+\bar{c} .
$$

Then, as a general condition, $g(x)$ evaluated at $(l, m, n)$ obtaining $g(l), g(m)$, and $g(n)$, is to be matched with $\bar{g}(x)$ at these data points as follows:

$$
\begin{aligned}
& g(l)=a l^{3}+b l^{2}+c l+d=\bar{a} l^{2}+\bar{b} l+\bar{c}, \\
& g(m)=a m^{3}+b m^{2}+c m+d=\bar{a} m^{2}+\bar{b} m+\bar{c}, \\
& g(n)=a n^{3}+b n^{2}+c n+d=\bar{a} n^{2}+\bar{b} n+\bar{c} .
\end{aligned}
$$

By solving for the coefficients of $\bar{g}(x)$, one obtains

$$
\begin{aligned}
\bar{g}(x)= & {[b+a(l+m+n)] x^{2}+[c-a(l m+m n+n l)] x } \\
& +(d+a l m n) .
\end{aligned}
$$

In order to obtain $(l, m, n)$ to define Eq. (7), the following three error-minimizing conditions are introduced:

Condition 1. One of three points is located at the value of $x$ corresponding to the maximum of the probability density function $\phi(x)$ of random variable $x$ :

$$
\begin{aligned}
& \text { Condition 2. } \int_{-\infty}^{\infty} g(x) \phi(x) d x=\int_{-\infty}^{\infty} \bar{g}(x) \phi(x) d x, \\
& \text { Condition 3. Minimize } E=\frac{1}{2}\left(\frac{\mu_{\varepsilon}}{\mu_{\min }}+\frac{s_{\varepsilon}}{s_{\min }}\right),
\end{aligned}
$$

where $\mu_{\varepsilon}=\int_{-\infty}^{\infty} \varepsilon(x) \phi(x) d x$ and $s_{\varepsilon}^{2}=\int_{-\infty}^{\infty} \varepsilon(x)^{2} \phi(x) d x$ are the first two moments of error function, $\varepsilon(x)=|g(x)-\bar{g}(x)|$, and $\mu_{\min }$ and $s_{\min }$ denote the minimum values of these moments, respectively.

Condition 1 indicates that one experimental point, which is usually $m$, should be located at the maximum likelihood point of $\phi(x)$. Condition 2 equates the first moment of $g(x)$ to that of $\bar{g}(x)$ as the mean of $g(x)$ is very important and provides a relationship between $l$ and $n$. Condition 3 requires that the mean and variance of the error function are minimized simultaneously and thus $l$ or $n$ is determined.

In this work all random variables are taken to follow normal distributions. Therefore, $(l, m, n)$ are determined as follows when a random variable follows the standard normal distribution with the mean $\mu=0$ and the standard deviation $\sigma=1$. By Condition 1 , the position, $m$, of the standard normal distribution is located at $m=\mu=0$. A term-by-term expansion of the integral in condition 2 followed by consideration of odd and even symmetries then gives

$$
l+n=0 .
$$

For convenience, let $l=m-k_{2} \sigma$ and $n=m+k_{1} \sigma$. Then Eq. (10) with $m=0$ becomes 


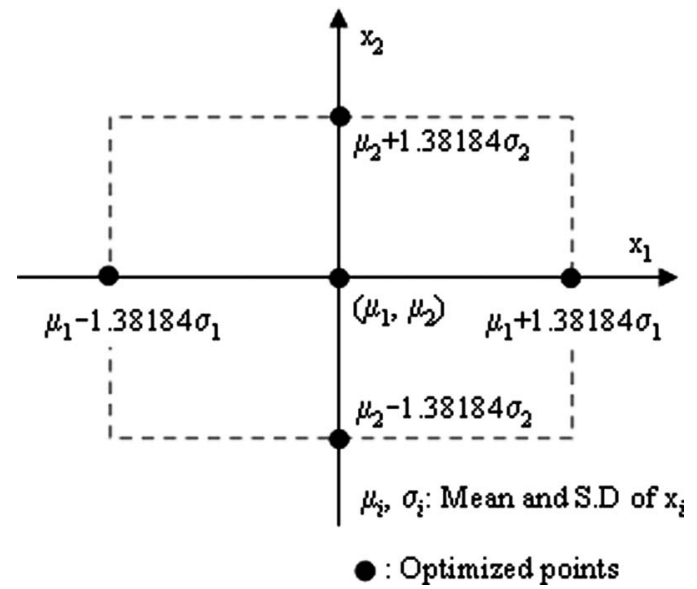

FIG. 1. Optimized positions of each variable and canonical experimental region in case of two random variables.

$$
k_{1}=k_{2} \text {. }
$$

The first two moments of the error function defined in Eq. (9) can be expressed as a function of only $k_{2}$ when using Eq. (11):

$$
\begin{aligned}
& \frac{\mu_{\varepsilon}}{|a|}=2 \times\left(-\frac{1}{\sqrt{2 \pi}}\left(-2 e^{-k_{2}^{2} / 2}+2-k_{2}^{2}\right)+\sqrt{\frac{2}{\pi}} e^{-k_{2}^{2} / 2}\right), \\
& \frac{s_{\varepsilon}}{|a|}=\sqrt{15-6 k_{2}^{2}+k_{2}^{4}} .
\end{aligned}
$$

The minimum values of the first two moments are $\mu_{\text {min }}$ $=1.1061|a|$ and $s_{\min }=\sqrt{6}|a|$ at $k_{2}=1.17741$ and $\sqrt{3}$, respectively. Condition 3 minimizing the sum of Eqs. (12) and (13) gives $k_{2}=1.38184$, thus from Eq. (11) $k_{1}=1.38184$. Therefore, the optimized distinct points are located at $(l, m, n)$ $=(m-1.38184 \sigma, m, m+1.38184 \sigma)$.

In case of $N$ random variables, the optimized points of each random variable are applied constructing an $N$-orthotope as illustrated in Fig. 1 for the case of two random variables. The center position of the $N$-orthotope matches point $\left(m_{1}, m_{2}, \ldots, m_{N}\right)$, and the locations of the sides along the axes are denoted by $l_{i}$ and $n_{i}$. Figure 1 shows the canonical experimental region when $x_{1}$ and $x_{2}$ follow the normal distributions with different standard deviations, respectively.

\section{Statistical moments of $\bar{g}(\mathbf{x})$ and probability}

After determining the canonical experimental region, it is uncomplicated to execute a design of experiments consid-

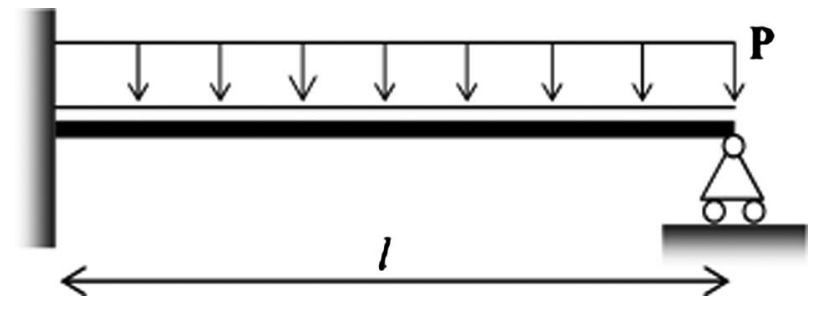

FIG. 2. Elastic beam with a uniform load $P$ (Ref. 9).
TABLE I. Parameters for random variables: Beam example.

\begin{tabular}{crl}
\hline \hline Random variable & \multicolumn{1}{c}{ Mean } & \multicolumn{1}{l}{ S.D. } \\
\hline$x_{1}(P)$ & $2 \mathrm{kN} / \mathrm{m}$ & $0.4 \mathrm{kN} / \mathrm{m}$ \\
$x_{2}(l)$ & $4 \mathrm{~m}$ & $0.4 \mathrm{~m}$ \\
$x_{3}\left(m_{F}\right)$ & $5 \mathrm{kN} \mathrm{m}$ & $0.4 \mathrm{kN} \mathrm{m}$ \\
\hline \hline
\end{tabular}

ering it; particularly a $D$-optimal design ${ }^{8}$ is used in this paper. It is also a straightforward process to evaluate a system response function at experimental points and to obtain the coefficients of an interpolating polynomial. The number of these is $(N+1)(N+2) / 2$ and the simplest procedure for their estimation is to use the same number of experimental points.

Once $\bar{g}(\mathbf{x})$ is constructed, the $k$ th moment of $\bar{g}(\mathbf{x})$ is easily and exactly obtained by the summation and multiplication of the moments of uncorrelated random variables, $x_{i}$ for $i=1$ to $N$, which are given from the probability density functions. Subsequently, probability distribution is obtained and the probability to satisfy $g(\mathbf{x}) \leqslant 0, \operatorname{Pr}[g(\mathbf{x}) \leqslant 0]$, is read from the distribution using the Pearson system. The Pearson system is an empirical system of frequency curves in which the probability density function of a random variable satisfies the following differential equation:

$$
\frac{1}{\phi(x)} \frac{d \phi(x)}{d x}=-\frac{a+x}{c_{0}+c_{1} x+c_{2} x^{2}},
$$

where the parameters are determined by the first four moments of $x$, and $\phi(x)$ is the probability density function of $x$. Various types of the probability density function of $x$ can be obtained from different relative values of the parameters. Detailed information about the Pearson system may be found in Ref. 7.

\section{Test example}

The test problem, which is referenced to ThoftChristensen and Baker, ${ }^{9}$ is about the failure condition of the maximum bending moment of an elastic beam with a uniform load shown in Fig. 2:

$$
g\left(P, l, m_{F}\right)=\frac{9}{128} P l^{2}-m_{F} \geqslant 0,
$$

where $P\left(x_{1}\right)$ is the uniform load, $m_{F}\left(x_{3}\right)$ is the critical limit moment, and $l\left(x_{2}\right)$ is the length. $P, m_{F}$ and $l$ are assumed uncorrelated random variables having the normal distributions with the mean and S.D. listed in Table I.

As explained in the previous section, the canonical experimental region, in step 2, is a cuboid whose center point is $\left(\mu_{P}, \mu_{l}, \mu_{m_{F}}\right)$ and the lengths of sides $\left(2 \times 1.38184 \sigma_{P}, 2\right.$ $\left.\times 1.38184 \sigma_{l}, 2 \times 1.38184 \sigma_{m_{F}}\right)$. In the next step, ten experi-

TABLE II. Resultant system response at ten experimental points.

\begin{tabular}{cccl}
\hline \hline$\left(x_{1}, x_{2}, x_{3}\right)$ & $g(\mathbf{x})$ & $\left(x_{1}, x_{2}, x_{3}\right)$ & \multicolumn{1}{c}{$g(\mathbf{x})$} \\
\hline$(1.4473,4.0,5.5527)$ & -3.9246 & $(2.5527,4.5527,4.4473)$ & -0.72692 \\
$(2.5527,3.4473,5.5527)$ & -3.4198 & $(2.5527,4.5527,5.5527)$ & -1.8324 \\
$(1.4473,3.4473,4.4473)$ & -3.2380 & $(2.0,4.5527,5.5527)$ & -2.6379 \\
$(1.4473,3.4473,5.5527)$ & -4.3435 & $(1.4473,4.5527,5.0)$ & -2.8908 \\
$(2.5527,3.4473,4.4473)$ & -2.3143 & $(2.0,4.0,5.0)$ & -2.75 \\
\hline \hline
\end{tabular}


TABLE III. Estimated moments of the resultant distribution and probability of failure.

\begin{tabular}{ccc}
\hline \hline & $\begin{array}{c}\text { Monte Carlo method } \\
(1000000)\end{array}$ & FAMM (10) \\
\hline Parameter & -2.7280 & -2.7266 \\
Mean & 0.7602 & 0.7625 \\
S.D. & 0.3187 & 0.3136 \\
Skewness & 3.2236 & 3.1795 \\
Kurtosis & $0.982 \times 10^{-3}$ & $0.977 \times 10^{-3}$ \\
$\operatorname{Pr}[g(\mathbf{x}) \geqslant 0]$ & & \\
\hline \hline
\end{tabular}

mental points from the $D$-optimal design are arranged as exhibited in Table II, including resultant system responses. The interpolating polynomial obtained from Table II is expressed as follows and coefficients of the sixth and seventh term are zero:

$$
\begin{aligned}
\bar{g}(\mathbf{x})= & -2.75+1.1434 X_{1}+1.1281 X_{2}-X_{3}+0.5569 X_{1} X_{2} \\
& +0.0 X_{1} X_{3}+0.0 X_{2} X_{3}+0.0111 X_{1}^{2}+0.1129 X_{2}^{2} \\
& +0.0222 X_{3}^{2},
\end{aligned}
$$

where $X_{1}=x_{1}-2.0, X_{2}=x_{2}-4.0$, and $X_{3}=x_{3}-5.0$.

The first four moments and the probability of failure from Eq. (16) are compared with the results from the Monte Carlo method using 1000000 observations. It is seen in Table III that they all agree very well with each other. In addition, the resultant distribution of FAMM becomes Pearson's type VI corresponding to a $\beta$ distribution. As shown in Fig. 3, the probability density function also agrees well with the histogram of the system response function estimated by the Monte Carlo method.

\section{DESCRIPTION OF THE $X Y$ STAGE AND FE ANALYSIS}

\section{A. Description of the $X Y$ stage}

The design requirements of the $X Y$ stage developed by Kang et al. have been $2 \mathrm{~mm}( \pm 1 \mathrm{~mm})$ working range, limited total size of $100 \mathrm{~mm} \times 100 \mathrm{~mm} \times 50 \mathrm{~mm}$, resolution less than $10 \mathrm{~nm}$, and minimum parasitic motion. ${ }^{6}$ Its structure is a stacked type such that the $Y$ stage is orthogonally piled up on

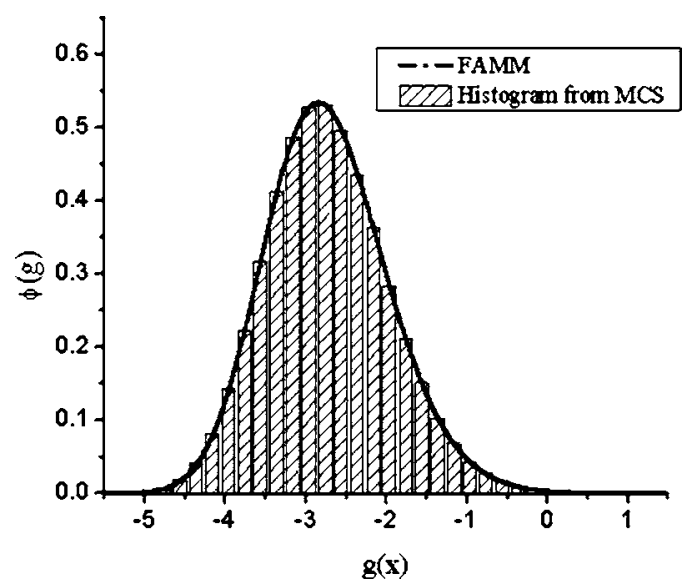

FIG. 3. Probability density function of the system response function [Pearson's types from FAMM (type VI) and histogram from the Monte Carlo method].

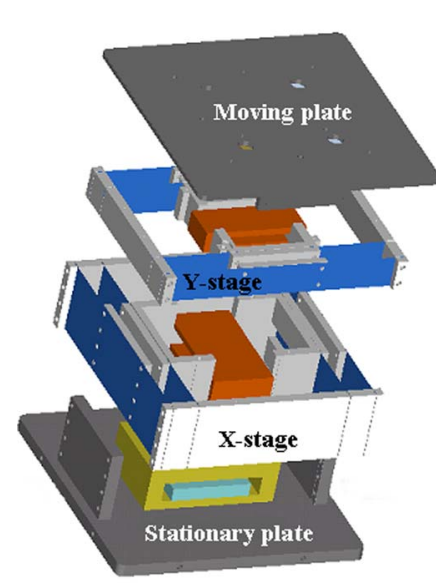

(a)

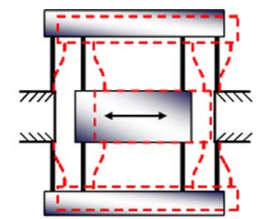

(b)

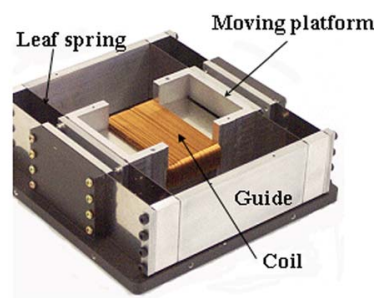

(c)
FIG. 4. (Color online) Description of the $X Y$ stage (Ref. 6): (a) CAD model; (b) concept design; (c) manufactured $X$ stage.

the $X$ stage as shown in Fig. 4(a). They have the same structure with a flexure (leaf-spring) guiding mechanism and a VCM actuator. The adopted flexure mechanism is composed of a double-compound linear spring. A supporting rectangular frame is fixed at two opposite sides with the stationary plate as shown in Fig. 4(b). Each stage is designed to have symmetry to remove parasitic movement. In addition, it provides high accuracy due to the characteristics of negligible backlash and stick-slip friction, and smooth and continuous motion. VCM also has inherently infinite resolution. Though a monolithic structure may be better suited, an assembly structure is used for this stage since it is difficult to make a small thickness monolithic leaf-spring guide, which is necessary for large travel range. Figure 4(c) shows the manufactured $X$ stage.

The main dimensions of each stage and material properties are summarized in Fig. 5 and Table IV, respectively. Resonant frequency that is in proportion to the response speed of the $X Y$ stage and working range are functions of the dimensions of leaf springs. The guide is composed of three different parts on each side and the three parts are clamped to each other and leaf springs as shown in Fig. 5. If all lengths of the six parts are exactly equal to the designed dimensions as shown in Fig. 5, no parasitic motion can occur. In reality, however, the $X Y$ stage is deformed after assemblage and the deformation may cause large parasitic motion during operation. Therefore, any variations from the designed dimensions cause deterioration of the performance functions of the $X Y$

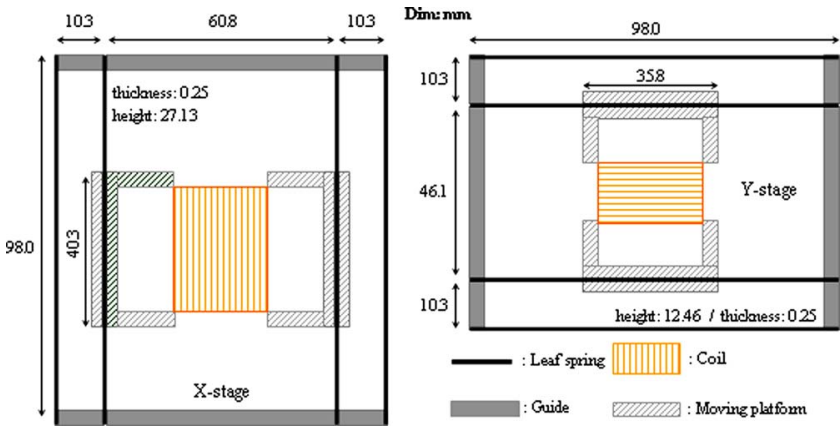

FIG. 5. (Color online) Main dimensions of the $X$ and $Y$ stage. 
TABLE IV. Material properties used for modeling the flexure stage.

\begin{tabular}{ccccc}
\hline \hline & Name & $\begin{array}{c}\text { Young's } \\
\text { modulus }(\mathrm{GPa})\end{array}$ & $\begin{array}{c}\text { Density } \\
\left(\mathrm{kg} / \mathrm{m}^{3}\right)\end{array}$ & $\begin{array}{c}\text { Poisson's } \\
\text { ratio }\end{array}$ \\
\hline $\begin{array}{c}\text { Leaf spring } \\
\text { Moving platform } \\
\text { and guide } \\
\text { Coil }\end{array}$ & Steel & 200 & 7850 & 0.29 \\
& Cluminum & 70 & 2710 & 0.33 \\
\hline \hline
\end{tabular}

stage. It is also noted that parasitic motions could still occur from inconsistencies in materials properties or clamping conditions, even if the lengths are exact, but these influences are not considered in this research.

\section{B. FE modeling and verification}

In order to investigate the variations of performance functions of the $X Y$ stage, a sound FE model is necessary and generated based on the dimensions in Fig. 5 and material properties in Table IV. The guiding mechanism is modeled by 1220 shell elements and the moving platforms and coils by 362 solid ones. In the process of generating the FE model, some simplifications are included: (1) the stationary plate in Fig. 4(a) is substituted by a displacement boundary condition as shown in Fig. 6; (2) the moving plate on the $Y$ stage in Fig. 4(a) is omitted and the corresponding mass is added to the clamping part as shown in Fig. 6, which is used to connect the leaf springs to it; (3) the coil whose shape is a hexahedron with a hole is simplified to be a plate with the same mass.

To verify the FE model of the $X Y$ stage, basic items such as mass and resonant frequencies are compared with measurements. A laser Doppler vibrometer (LDV) (Polytec. OFV 501 , OFV 3001) with a minimum resolution of $2 \mathrm{~nm}$ is used to measure the position. Measurements of the frequency response characteristics using a sine swept mode of a dynamic signal analyzer (Hewlett Packard 35 670A) are executed and the results are shown in Fig. 7. At the same time, the FE

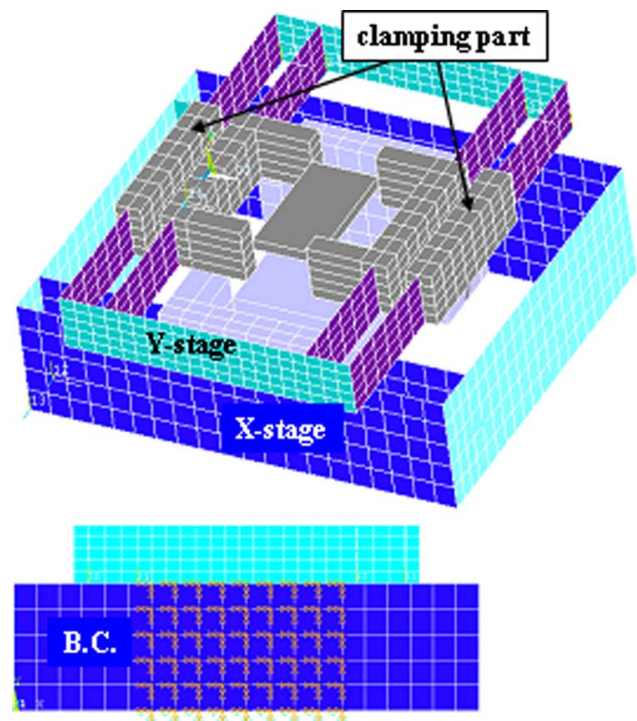

FIG. 6. (Color online) Finite element model of the $X Y$ stage and displacement boundary condition.

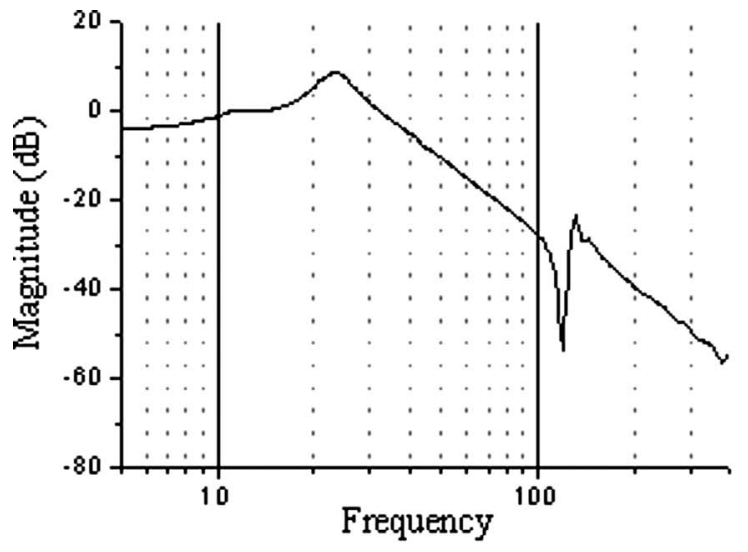

(a)

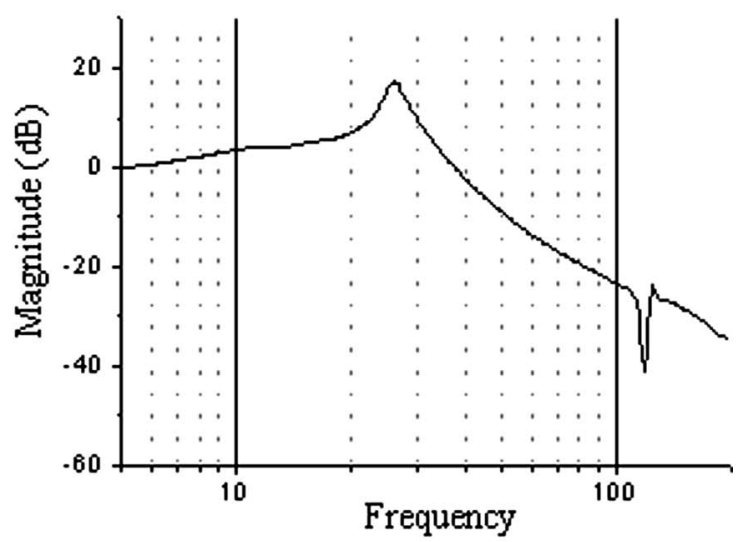

(b)

FIG. 7. Experimental results of the $X Y$ stage (FRF): (a) $X Y(23.93 \mathrm{~Hz})$; (b) only $Y(26.38 \mathrm{~Hz})$.

analysis is executed using the commercial code, ANSYS, and the mode shapes of each resonant frequency are shown in Fig. 8.

The results of mass and resonant frequencies are summarized in Table V. The masses of the model have errors of about $2.8 \%$ and $-3.0 \%$ for the $X Y$ stage and the $Y$ stage, and subsequently the errors of the first and second natural frequencies are about $4.0 \%$ and $11 \%$, respectively. The latter errors result from the difference of FE model and manufactured one. Especially, the thickness of leaf springs manufactured is not $0.25 \mathrm{~mm}$ but $0.24 \mathrm{~mm}$. If the thickness in FE model is assumed to be $0.24 \mathrm{~mm}$, the resonant frequencies are $23.98 \mathrm{~Hz}(+0.2 \%)$ and $27.51 \mathrm{~Hz}(+4.77 \%)$, respectively. Therefore, the FE model is taken acceptable for further analysis.

The degrees of freedom of the FE model are 10,212 and therefore, it requires large computational effort. In addition, the $X Y$ stage is a stacked type that the $Y$ stage is designed to be orthogonally piled up on the $X$ stage. Therefore, instead of the full model, we have taken the model of only the $X$ stage, which has a resonant frequency of $37.14 \mathrm{~Hz}$.

\section{DEFORMATION OF ASSEMBLY}

If all parts composed of the $X$ stage were manufactured without any fabrication error and assembled, the $X$ stage 


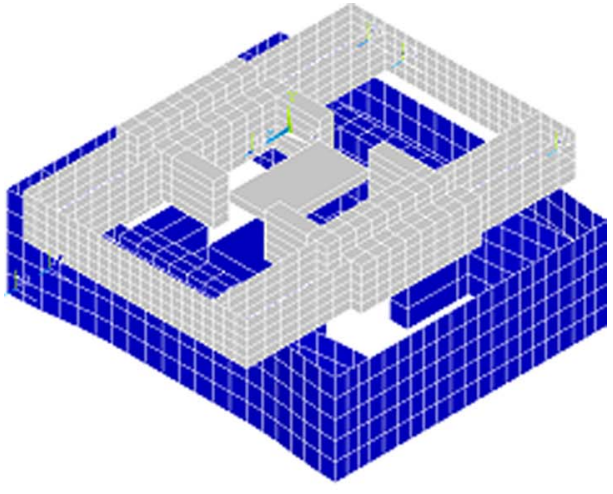

(a)

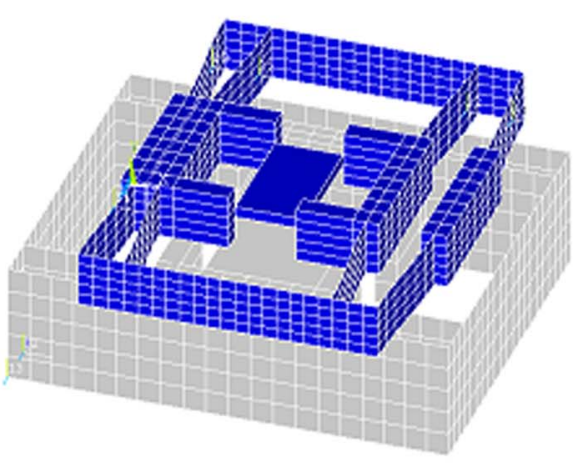

(b)

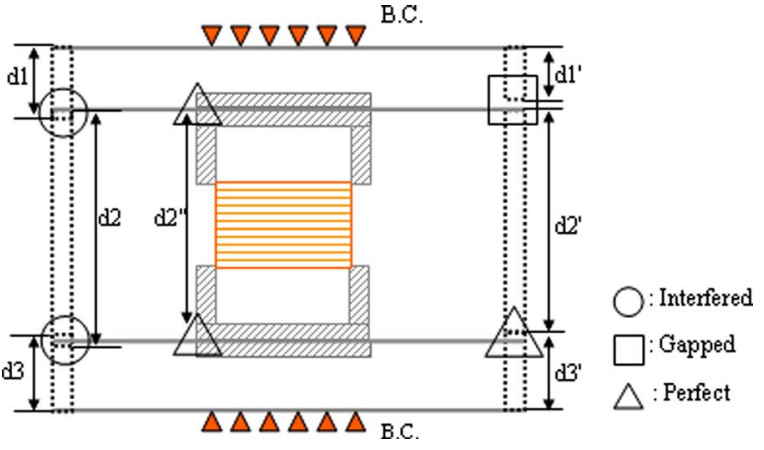

FIG. 9. (Color online) Effect of tolerances for six length dimensions.

are specified, the others are automatically determined by FE analysis. Here the three conditions are part of design variables. There are 180 design variables because there are six nodes at CA1 and CA3, respectively, and 48 nodes at CA2 and thus $x, y$, and $z$ locations of 60 nodes are the design variables. To reduce the number of design variables, an imaginary contacting area is considered as shown in Fig. 10(b) and the number of design variables becomes 48 because there are only four nodes at CA2.

Consider a test problem of finding the initial deformation of the $X$ stage, which is assumed to have fabrication errors as shown in Table VI and to have no errors in other dimensions.

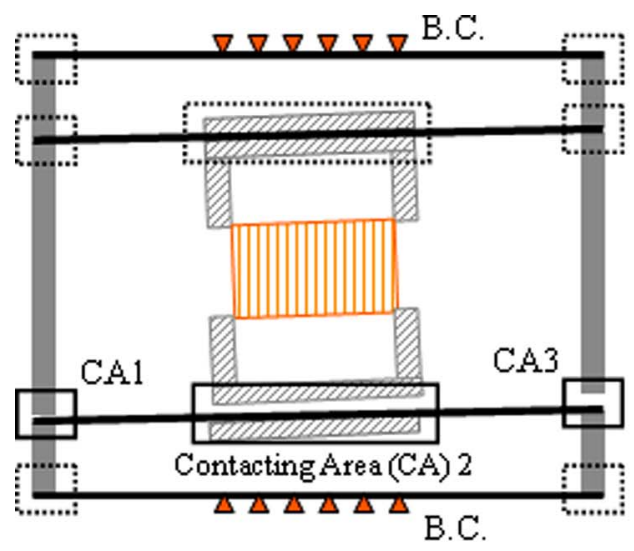
are not known, but it is expected that the equilibrium state of the assembled $X$ stage has the minimum of strain energy. The deformed shape is thus found by minimizing the strain energy.

The minimum is taken among all possible matching conditions among components, that is, the locations of the nodes to be bonded in the contacting areas (CA). There are a total of ten contacting areas as shown in Fig. 10(a) where the boundary conditions are unknown. However, if displacement conditions at the three boxes with a solid line in Fig. 10(a)

TABLE V. Comparison between measurements and FE calculations.

\begin{tabular}{cccc}
\hline \hline & & Measurements & FE calculations \\
\hline \multirow{2}{*}{ Mass } & $X Y$ & $316.3 \mathrm{~g}$ & $324.82 \mathrm{~g}(+2.8 \%)$ \\
& Only $Y$ & $138.13 \mathrm{~g}$ & $135.38 \mathrm{~g}(-3.0 \%)$ \\
\multirow{2}{*}{ Resonant } & $\mathrm{f}_{1}$ & $23.93 \mathrm{~Hz}$ & $25.46 \mathrm{~Hz}(+4.0 \%)$ \\
frequency & $f_{2}$ & $26.38 \mathrm{~Hz}$ & $29.21 \mathrm{~Hz}(+11 \%)$ \\
\hline \hline
\end{tabular}

(a)

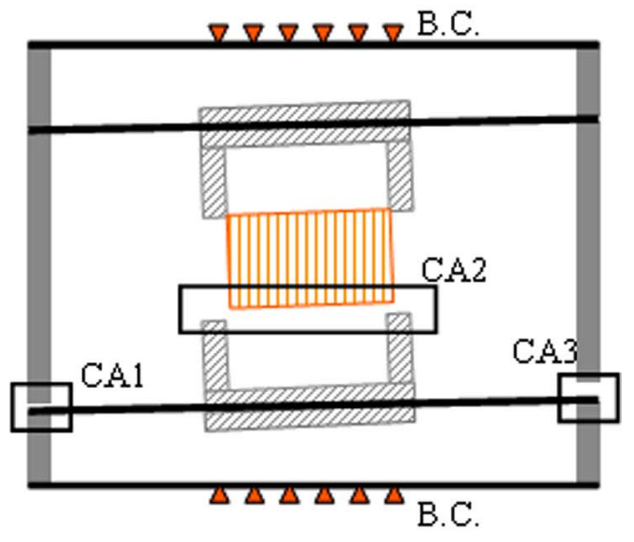

(b)
FIG. 10. (Color online) Definition of design variables at: (a) Real contacting areas indicated by the boxes; (b) imaginary contacting areas. 
TABLE VI. Definition of a test problem of finding residual deformation.

\begin{tabular}{cccccccc}
\hline \hline Dimensions (mm) & $d 1$ & $d 1^{\prime}$ & $d 2$ & $d 2^{\prime}$ & $d 2^{\prime \prime}$ & $d 3$ & $d 3^{\prime}$ \\
\hline Designed & 10.3 & 10.3 & 60.8 & 60.8 & 60.8 & 10.3 & 10.3 \\
Manufactured & 10.35 & 10.25 & 60.8 & 60.75 & 60.8 & 10.35 & 10.3 \\
\hline \hline
\end{tabular}

The notations are shown in Fig. 9. In the minimization, the gradient of strain energy are required and calculated by a central difference method with $0.1 \%$ perturbation. A Broyden, Fletcher, Goldfarb, and Shanno (BFGS) algorithm ${ }^{10}$ is utilized. The objective function or the strain energy is dramatically reduced from $761.0 \times 10^{-5} \mathrm{~J}$ to $3.03 \times 10^{-5} \mathrm{~J}$ in ten iterations and $501 \mathrm{FE}$ calculations. The contours of von Mises strain show that it is distributed very evenly on all leaf springs as shown in Fig. 11. The small fabrication errors listed in Table VI have caused much smaller deformation in the assembly when compared with the total size of the $X$ stage. However, this apparently small deformation can deteriorate the system performance, especially causing a large parasitic motion due to breakage of the symmetry.

\section{STATISTICS OF SYSTEM PERFORMANCE UNDER TOLERANCES}

Important design requirements of the stage are high displacement resolution, wide working range, high-speed response, and minimum parasitic motion. The flexure guiding mechanism and VCM are suitable for high displacement resolution and no parasitic motion as mentioned in Sec. III. As explained before, however, the accumulative tolerances, especially in the guide body, cause residual deformation after assembling. This leads to parasitic motion during operation, to be defined as the ratio of $x$ - to $y$ - axis displacement at the central position of the coil part, $d_{y} / d_{x}$. Response speed and working range are largely dependent on the thickness of leaf springs. Since the range is much dependent on VCM, it is not included in our study here. The response speed is treated in terms of natural frequency. Therefore, in this paper, we aim to evaluate the statistical moments, the probability density functions, and the probability of satisfaction about parasitic motion and resonant frequency.

As mentioned above, the two system responses are primarily functions of the thickness of leaf springs and the six

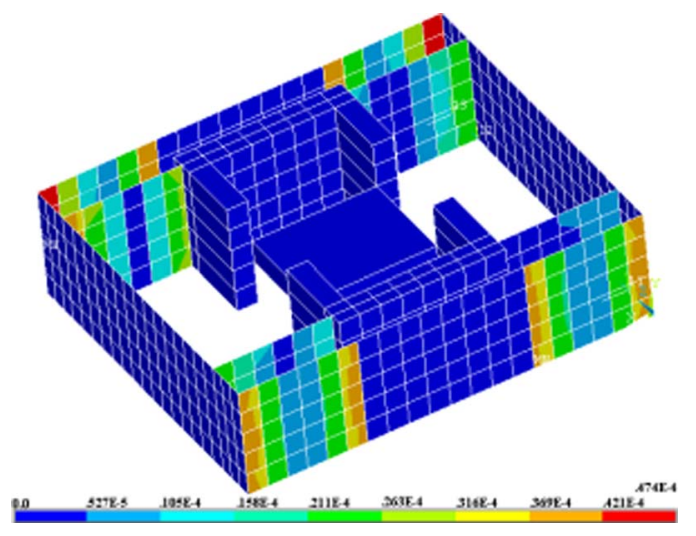

FIG. 11. (Color online) Contour of von Mises strain at equilibrium state. length dimensions in the guide. The random variables associated with these dimensions are reasonably assumed to follow the normal distributions whose standard deviations are equal to tolerances divided by 3.0 , as shown in Table VII. The influences of tolerances are studied using the function approximation moment method. FAMM, as explained in Sec. II, approximates the two implicit system performance functions, that is, parasitic motion and resonant frequency with two quadratic interpolating polynomials. The statistical moments are then obtained from the approximate functions as summarized in Table VIII. The resulting polynomials are not given here because they have many terms.

In order to generate them, FAMM constructs the canonical experimental region as defined in Sec. II and then 45 experimental points are determined by the $D$-optimality criterion considering it. The system responses-natural frequency and $d_{y}$ and $d_{x}$ in modal and static analysis, respectively_are calculated at each experimental point. In the latter case, an electromagnetic force, $10 \mathrm{~N}$, is assumed to be applied to the coil. To obtain these responses, residual deformation corresponding to each experimental point after assemblage is calculated using the minimization process explained earlier. Therefore, the total number of FE calculations for each response function is around 500 for each experimental point and the total computational cost is rather large. Finally, the Pearson system is utilized to obtain the probability density functions based on the first four moments listed in Table VIII and the probabilities of satisfaction about system requirements are calculated. The resulting distributions of parasitic motion and natural frequency are found to be of types I and IV of the Pearson system, respectively, as shown in Fig. 12.

The mean of the first natural frequency is $37.15 \mathrm{~Hz}$ and the standard deviation is $1.45 \mathrm{~Hz}$, which is rather small. We thus conclude that the effect of tolerances on response speed is insignificant. However, the mean of parasitic motion is $2.04 \mathrm{~nm}$ below $10 \mathrm{~nm}$ displacement resolution when the stage reaches the peak working range of $1 \mathrm{~mm}$, but its standard deviation is $104.7 \mathrm{~nm}$, which is rather huge. Figure

TABLE VII. Definition of random variables: $X$ stage (refer to the Fig. 9).

\begin{tabular}{ccc}
\hline \hline Dimension & Mean $(\mathrm{mm})$ & S.D. $(\mathrm{mm})$ \\
\hline$d 1\left(x_{1}\right)$ & 10.3 & $0.050 / 3.0$ \\
$d 1^{\prime}\left(x_{2}\right)$ & 10.3 & $0.050 / 3.0$ \\
$d 2\left(x_{3}\right)$ & 60.8 & $0.050 / 3.0$ \\
$d 2^{\prime}\left(x_{4}\right)$ & 60.8 & $0.050 / 3.0$ \\
$d 2^{\prime \prime}\left(x_{5}\right)$ & 60.8 & $0.050 / 3.0$ \\
$d 3\left(x_{6}\right)$ & 10.3 & $0.050 / 3.0$ \\
$d 3^{\prime}\left(x_{7}\right)$ & 10.3 & $0.050 / 3.0$ \\
thickness $\left(=x_{8}\right)$ & 0.25 & $0.020 / 3.0$ \\
\hline \hline
\end{tabular}


TABLE VIII. Statistical moments of two performance functions of the $X$ stage.

\begin{tabular}{ccc}
\hline \hline & $d_{y} / d_{x}$ (Parasitic motion) & $f_{1}$ (Natural frequency) $(\mathrm{Hz})$ \\
\hline Mean & $2.004 \times 10^{-6}$ & 37.1519 \\
S.D. & $1.047 \times 10^{-4}$ & 1.4526 \\
Skewness & -0.05518 & 0.03566 \\
Kurtosis & 4.94023 & 3.0017 \\
\hline \hline
\end{tabular}

12(a) shows the probability of satisfaction about $d_{y} / d_{x}$, that is, the probability to satisfy the system requirement, $-10 \mathrm{~nm} / 1 \mathrm{~mm} \leqslant d_{y} / d_{x} \leqslant 10 \mathrm{~nm} / 1 \mathrm{~mm}$, is $8.66 \%$. This indicates that this guiding mechanism has trouble in controlling parasitic motions due to uncertainties from tolerances.

In order to resolve this problem, three methods may be possible: (1) Reduce the tolerances allocated at six parts in the guide; (2) install a tracking system and a compensator in the stage; (3) introduce a process to measure six parts in the guide and to do additional work for correction. The first method has some limitation on reducing the standard deviation of parasitic motion below the displacement resolution because the allocated tolerances are already small. This, however, requires further study of tolerance allocation. The second makes the system complex and expensive. Furthermore, a double-compound linear spring itself becomes meaningless because it is used to remove parasitic motion. In order to obtain a hint of the third method, the amounts of parasitic motion, $d_{y} / d_{x}$, at each experimental point are carefully analyzed, and the results are classified into three groups according to $d_{y} / d_{x}$. Table IX shows three typical examples. They indicate that the differences between $d 1$ and $d 1^{\prime}$, and $d 3$ and $d 3^{\prime}$, respectively, have a larger effect on $d_{y} / d_{x}$ than the absolute dimensions of them. Therefore, the observations recommend that they should be minimized although an additional process is introduced. The process may be to measure all parts in the guide to pair up or to do additional finishing work.

\section{CONCLUSION}

The function approximation moment method (FAMM) is developed and the influence of tolerance on various response functions of a precision stage, especially parasitic motion and resonant frequency, is studied. The function approximation in FAMM is performed by an interpolating polynomial of order 2 constructed on a canonical experimental region, which is determined by three error-minimizing conditions for better accuracy. The unknown coefficients of the polynomial are estimated by a $D$-optimal design. The four moments from

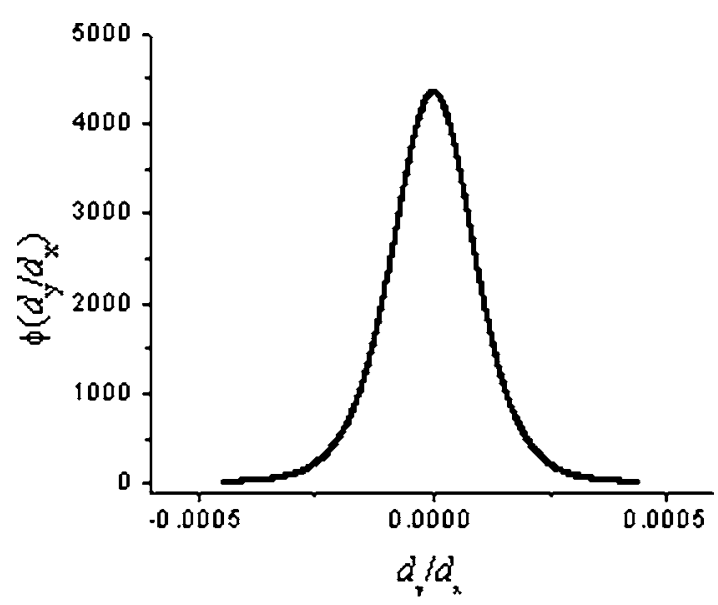

(a)

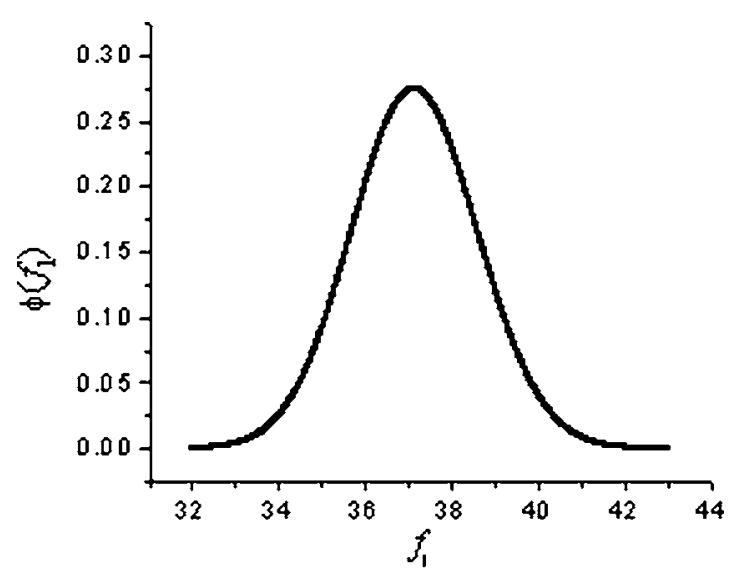

(b)

FIG. 12. Probability density functions estimated by the Pearson system: (a) Parasitic motion (type I); (b) natural frequency (type IV).

the interpolating polynomial and subsequently the probability of a given condition are obtained without additional efforts and time.

A finite element model of the $X Y$ stage is generated and verified with basic measurements. The stage, which is nonmonolithic, may be deformed after assemblage because of accumulative tolerances. The possible deformation is found by the formulation of minimizing strain energy. The response functions such as resonant frequency and parasitic motion are evaluated based on the FE model with the residual deformation. Their results at 45 experimental points are utilized for FAMM to estimate the first four moments, the probability density functions, and the probability of satisfaction. It is shown that parasitic motion is quite sensitive to uncertainties

TABLE IX. Parasitic motion corresponding to different experimental points.

\begin{tabular}{ccccccccc}
\hline \hline & \multicolumn{8}{c}{ Perturbations of each dimension $(\mathrm{mm})$} \\
\cline { 2 - 7 } & $d 1$ & $d 1^{\prime}$ & $d 2$ & $d 2^{\prime}$ & $d 2^{\prime \prime}$ & $d 3$ & $d 3^{\prime}$ & \\
\hline Group I & -0.023 & -0.023 & -0.023 & +0.023 & -0.023 & +0.023 & +0.023 & 0.12 \\
Group II & -0.023 & +0.023 & +0.023 & +0.023 & -0.023 & -0.023 & -0.023 & -27.3 \\
Group III & -0.023 & +0.023 & -0.023 & +0.023 & -0.023 & 0.0 & -0.023 & -146.7 \\
\hline \hline
\end{tabular}


due to tolerances. It is recommended that without reducing tolerances this motion can be made small if the differences between dimensions $d 1$ and $d 1^{\prime}$, and $d 3$ and $d 3^{\prime}$, respectively, are minimized through additional processes such as component pairing and finishing before assemblage. The methods and finite element modeling for residual deformation developed here are shown very efficient to characterize the performance variations due to tolerances in terms of probability density functions.

${ }^{1}$ S. T. Smith, D. G. Chetwynd, and D. K. Bowen, J. Phys. E 20, 977 (1988).

${ }^{2}$ J. W. Ryu and D. G. Gweon, Precis. Eng. 21, 83 (1997).
${ }^{3}$ R. E. Melchers, Structural Reliability Analysis and Prediction (Wiley, New York, 1999).

${ }^{4}$ Y. G. Zhao and T. Ono, Struct. Safety 23, 47 (2001).

${ }^{5}$ H. S. Seo and B. M. Kwak, Int. J. Prod. Res. 40, 931 (2002).

${ }^{6}$ D. W. Kang, D. M. Kim, K. H. Kim, J. Y. Shim, and D. G. Gweon, ASPE 2003 Annual Meeting, Portland, OR, p. 183 (2003).

${ }^{7}$ N. L. Johnson, S. Kotz, and N. Balakrishnan, Continuous Univariate Distributions (Wiley, New York, 1994).

${ }^{8}$ R. H. Myers and D. C. Montgomery, Response Surface Methodology: Process and Product Optimization Using Designed Experiments (Wiley, New York, 1995).

${ }^{9}$ P. Thoft-Christensen and M. J. Baker, Structural Reliability Theory and its Applications (Springer, Berlin, 1982).

${ }^{10}$ A. D. Belegundu and T. R. Chandrupatla, Optimization Concepts and Applications in Engineering (Prentice-Hall, New Jersey, 1999). 